\title{
ASYMPTOTIC SOLUTION OF THE PERTURBED FIRST BOUNDARY VALUE PROBLEM WITH A NON-SMOOTH COEFFICIENT
}

\author{
D.A. Tursunov, M.O. Orozov \\ Osh State University, Osh, Kyrgyz Republic \\ E-mail:d osh@rambler.ru
}

In this paper, we consider the first boundary value problem, that is the Dirichlet problem in a ring for a linear inhomogeneous second-order elliptic equation with two independent variables containing a small parameter in front of the Laplacian. The equation potential is not a smooth function in the field under study. There exists a unique solution of the first boundary value problem under consideration. It is impossible to construct an obvious solution of the first boundary value problem. We are interested in the influence of the small parameter on the solution of the Dirichlet problem in the field under study when the small parameter tends to zero. That is why we need to construct an asymptotic solution of the first boundary value problem in a ring. The problem under consideration has two singularities (a bisingular problem): presence of a small parameter in front of the Laplacian, and solution of a relevant unperturbed equation is not a smooth function in the field under study. To construct an asymptotic solution, we use a modified method of boundary functions since it is impossible to use a classical method of boundary functions. To begin with, we construct a formal asymptotic solution as per the small parameter, and then we evaluate the remainder term of the asymptotic expansion. As a result, we have constructed complete uniform asymptotic expansion of the first boundary value problem in a ring as per the small parameter. The constructed series of the solution of the first boundary value problem is asymptotic in the sense of Erdey.

Keywords: Dirichlet problem for a ring; bisingular problem; asymptotics; Laplace operator; small parameter.

Consider the partial differential equation of elliptic type

$$
\varepsilon \Delta u(\rho, \varphi, \varepsilon)-\sqrt{\rho-1} u(\rho, \varphi, \varepsilon)=f(\rho, \varphi), \quad(\rho, \varphi) \in D,
$$

with the inhomogeneous boundary conditions of the first kind

$$
u(1, \varphi, \varepsilon)=\psi_{1}(\varphi), u(\mathrm{a}, \varphi, \varepsilon)=\psi_{2}(\varphi), \varphi \in[0,2 \pi]
$$

where $0<\varepsilon$ is a small parameter, $\Delta=\frac{\partial^{2}}{\partial \rho^{2}}+\frac{1}{\rho} \frac{\partial}{\partial \rho}+\frac{1}{\rho^{2}} \frac{\partial^{2}}{\partial \varphi^{2}}, f \in C^{\infty}(\bar{D}), D=\{(\rho, \varphi) \mid 1<\rho<a$ is a constant, $0 \leq \varphi \leq 2 \pi\}, \psi_{k} \in C^{\infty}[0,2 \pi], k=1,2$.

Mathematical models of many natural phenomena are described using boundary value problems for partial differential equations [1-2].

According to the theory of partial differential equations, the solution to considered first boundary value problem (2) for differential equation of elliptic type (1) exists and is unique [3]. We are interested in the behavior of the solution, i. e. dependence of this solution on the small parameter $\varepsilon$, where $\varepsilon \rightarrow 0$. We consider the question about the part of the domain $\bar{D}$ in which passage to the limit is performed.

The considered first boundary value problem has two features (bisingularity) [2]. The first singularity is the fact that the solution to the limit equation $(\varepsilon=0)$ cannot satisfy the boundary conditions, since the limit equation is not a differential equation. The second feature states that the solution to the limit equation is not a smooth function in the domain $\bar{D}$ :

$$
u(\rho, \varphi, 0)=-f_{0}(\rho, \varphi) / \sqrt{\rho-1} .
$$

In order to show how this nonsmooth solution affects the asymptotic behavior of the solution to the Dirichlet problem, we consider the classical outer asymptotic expansion of the solution to the first boundary value problem: 


$$
U(\rho, \varphi, \varepsilon)=\sum_{k=0}^{\infty} \varepsilon^{k} u_{k}(\rho, \varphi), \varepsilon \rightarrow 0 .
$$

Substitute series (3) into differential equation of elliptic type (1) and equate the coefficients at the same powers of $\varepsilon$, then we obtain:

$$
-\sqrt{\rho-1} u_{0}(\rho, \varphi)=f(\rho, \varphi), \quad \sqrt{\rho-1} u_{k}(\rho, \varphi)=\Delta u_{k-1}(\rho, \varphi), \mathrm{k} \in \mathrm{N} .
$$

Here we determine all $u_{k}(\rho, \varphi)$ as follows: $u_{0}(\rho, \varphi)=-\frac{f(\rho, \varphi)}{\sqrt{\rho-1}}, \quad u_{k}(\rho, \varphi)=\frac{\Delta u_{k-1}(\rho, \varphi)}{\sqrt{\rho-1}}, k \in N$.

In the general case, all these functions $u_{k}(\rho, \varphi)$ have increasing singularities of the form

$$
u_{k}(\rho, \varphi)=O\left((\rho-1)^{-(5 k+1) / 2}\right), \rho \rightarrow 1, k \in N_{0}=0,1,2, \ldots
$$

Therefore, asymptotic solution (3) can be represented as follows:

$$
U(\rho, \varphi, \varepsilon)=\frac{1}{\sqrt{\rho-1}} \sum_{k=0}^{\infty}\left(\frac{\varepsilon}{\sqrt{(\rho-1)^{5}}}\right)^{k} F_{k}(\rho, \varphi), \varepsilon \rightarrow 0
$$

where $F_{k} \in C^{\infty}(\bar{D}), k=0,1,2, \ldots$

Hence, series (3) is an asymptotic solution to the first boundary value problem only in the domain $\left\{(\rho, \varphi) \mid 1+\varepsilon^{2 / 5}<\rho<a, 0 \leq \varphi \leq 2 \pi\right\}$, and does not satisfy the boundary condition $u(a, \varphi, \varepsilon)=\psi_{2}(\varphi)$ on the circle $\rho=a$, and solution (3) loses its asymptotic character in the domain $\left\{(\rho, \varphi) \mid 1 \leq \rho \leq 1+\varepsilon^{2 / 5}\right.$, $0 \leq \varphi \leq 2 \pi\}$.

Let us prove the following theorem.

Theorem. The asymptotic solution to first boundary value problem (1) and (2) can be represented in the following form:

$$
u(\rho, \varphi, \varepsilon)=\sum_{k=0}^{\infty} \varepsilon^{k} v_{k}(\rho, \varphi)+\sum_{k=0}^{\infty} \lambda^{k} z_{k}(t, \varphi)+\frac{1}{\mu} \sum_{k=0}^{\infty} \mu^{k} w_{k}(\tau, \varphi), \varepsilon \rightarrow 0,
$$

where $t=(\mathrm{a}-\rho) / \lambda, \lambda=\sqrt{\varepsilon}, \tau=(\rho-1) / \mu^{2}, \mu=\sqrt[5]{\varepsilon}$.

The functions $v_{k}(\rho, \varphi), \mathrm{z}_{k}(t, \varphi), w_{k}(\tau, \varphi)$ are specified below, in the proof of Theorem.

Proof. First, construct a formal asymptotic solution to the first boundary value problem. As usual, we look for such a solution in the form [4-6]

$$
u(\rho, \varphi, \varepsilon)=V(\rho, \varphi, \varepsilon)+Z(\mathrm{t}, \varphi, \lambda)+\mathrm{W}(\tau, \varphi, \mu),
$$

where $\quad V(\rho, \varphi, \varepsilon)=\sum_{k=0}^{\infty} \varepsilon^{k} v_{k}(\rho, \varphi), \quad Z(t, \varphi, \lambda)=\sum_{k=0}^{\infty} \lambda^{k} z_{k}(t, \varphi), \quad t=(\mathrm{a}-\rho) / \lambda, \lambda=\sqrt{\varepsilon}$, $W(\tau, \varphi, \mu)=\frac{1}{\mu} \sum_{k=0}^{\infty} \mu^{k} w_{k}(\tau, \varphi), \tau=(\rho-1) / \mu^{2}, \mu=\sqrt[5]{\varepsilon}$.

Taking into account conditions (2), we obtain:

$$
\begin{gathered}
W(0, \varphi, \mu)=\psi_{1}(\varphi)-V\left(1, \varphi, \mu^{5}\right), \forall k: \lim _{\tau \rightarrow \infty} w_{k}(\tau, \varphi)=0, \varphi \in[0,2 \pi] ; \\
Z(0, \varphi, \lambda)=\psi_{2}(\varphi)-V\left(a, \varphi, \lambda^{2}\right), \forall k: \lim _{t \rightarrow \infty} z_{k}(t, \varphi)=0, \varphi \in[0,2 \pi] .
\end{gathered}
$$

We write differential equation of elliptic type (1) in the form [4-6]

$$
\varepsilon \Delta u(\rho, \varphi, \varepsilon)-\sqrt{\rho-1} u(\rho, \varphi, \varepsilon)=f(\rho, \varphi, \varepsilon)-h(\rho, \varphi, \varepsilon)+h(\rho, \varphi, \varepsilon),
$$

where $h(\rho, \varphi, \varepsilon)=\sum_{k=0}^{\infty}\left(\varepsilon^{2 k} h_{2 k}(\rho, \varphi)+\varepsilon^{2 k+1}\left(\frac{h_{2 k+1,1}(\rho, \varphi)}{\sqrt{\rho-1}}+\frac{h_{2 k+1,3}(\varphi)}{\sqrt{(\rho-1)^{3}}}\right)\right), h_{2 k+1,1}(\rho, \varphi), h_{2 k+1,3}(\varphi)$ are unknown functions for the present.

Substituting formal series (4) into differential equation of elliptic type (7), we have

$$
\varepsilon \Delta V(\rho, \varphi, \varepsilon)-\sqrt{\rho-1} V(\rho, \varphi, \varepsilon)=f(\rho, \varphi, \varepsilon)-h(\rho, \varphi, \varepsilon),(\rho, \varphi) \in D,
$$




$$
\begin{gathered}
\mu\left(\frac{\partial^{2} W}{\partial \tau^{2}}+\mu^{2} c \frac{\partial W}{\partial \tau}+\left(\mu^{2} c\right)^{2} \frac{\partial^{2} W}{\partial \varphi^{2}}-\sqrt{\tau} W\right)=h\left(1+\tau \mu^{2}, \varphi, \mu^{5}\right),(\tau, \varphi) \in D_{0}, \\
\frac{\partial^{2} Z}{\partial t^{2}}-\lambda \tilde{c} \frac{\partial Z}{\partial t}+(\lambda \tilde{c})^{2} \frac{\partial^{2} Z}{\partial \varphi^{2}}-\sqrt{a-1} \sqrt{1-\frac{\lambda t}{a-1}} Z=0,(t, \varphi) \in D_{1},
\end{gathered}
$$

where $\quad c=\frac{1}{1+\tau \mu^{2}}, \frac{1}{a} \leq c \leq 1, \quad D_{0}=\left\{(\tau, \varphi) \mid 0<\tau<(a-1) / \mu^{2}, 0<\varphi \leq 2 \pi\right\}, \quad D_{1}=\{(t, \varphi) \mid 0<t<(a-$ 1) $/ \lambda, 0<\varphi \leq 2 \pi\}, \tilde{c}=\frac{1}{a-\lambda t}, \frac{1}{a} \leq \tilde{c} \leq 1, W=W(\tau, \varphi, \mu), Z=Z(t, \varphi, \lambda)$.

From (8), taking into account that $V(\rho, \varphi, \varepsilon)=\sum_{k=0}^{\infty} \varepsilon^{k} v_{k}(\rho, \varphi)$ and equating the coefficients at the same degrees of $\varepsilon$, we obtain:

$$
v_{0}(\rho, \varphi)=-\frac{f(\rho, \varphi)-h_{0}(\varphi)}{\sqrt{\rho-1}}
$$

if $h_{0}(\varphi)=f(1, \varphi)$, then $v_{0} \in C(\bar{D})$.

In this case, $v_{0}(\rho, \varphi)=-\sqrt{\rho-1} \tilde{v}_{0}(\rho, \varphi), \quad \tilde{v}_{0} \in C^{\infty}(\bar{D})$ and

$$
\Delta v_{0}=\frac{1}{4(\rho-1)^{3 / 2}} \tilde{v}_{0}-\frac{1}{\sqrt{\rho-1}} \frac{\partial \tilde{v}_{0}}{\partial \rho}-\sqrt{\rho-1} \frac{\partial^{2} \tilde{v}_{0}}{\partial \rho^{2}}-\frac{1}{\rho}\left(\frac{\tilde{v}_{0}}{2 \sqrt{\rho-1}}+\sqrt{\rho-1} \frac{\partial \tilde{v}_{0}}{\partial \rho}\right)-\sqrt{\rho-1} \frac{\partial^{2} \tilde{v}_{0}}{\partial \varphi^{2}} .
$$

Let us determine $v_{1}(\rho, \varphi)$ :

$$
v_{1}(\rho, \varphi)=\frac{\Delta v_{0}(\rho, \varphi)+h_{1}(\rho, \varphi)}{\sqrt{\rho-1}},
$$

let $h_{1}(\rho, \varphi)=-\frac{\tilde{v}_{0,0}(\varphi)}{4(\rho-1)^{3 / 2}}+\frac{3 \tilde{v}_{0,1}(\varphi) \rho-2 \tilde{v}_{0,0}(\varphi)}{4 \rho \sqrt{\rho-1}}$, where $\tilde{v}_{0,0}(\varphi)=\tilde{v}_{0}(1, \varphi), \tilde{v}_{0,1}(\varphi)=\frac{\partial \tilde{v}_{0}(1, \varphi)}{\partial \rho}$.

Then

Hence,

$$
\Delta v_{0}(\rho, \varphi)+h_{1}(\rho, \varphi)=\sqrt{\rho-1} \tilde{\tilde{v}}_{0}(\rho, \varphi), \tilde{\tilde{v}}_{0} \in C^{\infty}(\overline{\mathrm{D}}) .
$$

$$
v_{1}(\rho, \varphi)=\frac{\Delta v_{0}(\rho, \varphi)+h_{1}(\rho, \varphi)}{\sqrt{\rho-1}}=\tilde{\tilde{v}}_{0}(\rho, \varphi) .
$$

Continuing this process in a similar way, for $v_{2 k+1}(\rho, \varphi)$ we have:

let

$$
v_{2 k+1}(\rho, \varphi)=\frac{\Delta v_{2 k}(\rho, \varphi)+h_{2 k+1}(\rho, \varphi)}{\sqrt{\rho-1}},
$$

where

$$
h_{2 k+1}(\rho, \varphi)=-\frac{\tilde{v}_{2 \mathrm{k}, 0}(\varphi)}{4(\rho-1)^{3 / 2}}+\frac{3 \tilde{v}_{2 k 0,1}(\varphi) \rho-2 \tilde{v}_{2 k, 0}(\varphi)}{4 \rho \sqrt{\rho-1}}
$$

then

$$
\tilde{v}_{2 k, 0}(\varphi)=\tilde{v}_{2 k}(1, \varphi), \tilde{v}_{2 k, 1}(\varphi)=\frac{\partial \tilde{v}_{2 k}(1, \varphi)}{\partial \rho},
$$

Hence, we have:

$$
\Delta v_{2 k}(\rho, \varphi)+h_{2 k+1}(\rho, \varphi)=\sqrt{\rho-1} \tilde{\tilde{v}}_{2 k}(\rho, \varphi), \tilde{\tilde{v}}_{2 k} \in C^{\infty}(\overline{\mathrm{D}}) .
$$

$$
v_{2 k+1}(\rho, \varphi)=\frac{\Delta v_{2 k}(\rho, \varphi)+h_{2 k+1}(\rho, \varphi)}{\sqrt{\rho-1}}=\tilde{\tilde{v}}_{2 k}(\rho, \varphi) .
$$

And, for $v_{2 k}(\rho, \varphi)$, we have: 


$$
v_{2 k}(\rho, \varphi)=\frac{\Delta v_{2 k-1}(\rho, \varphi)+h_{2 k}(\rho, \varphi)}{\sqrt{\rho-1}},
$$

if $h_{2 k}(\varphi)=-\Delta v_{2 k-1}(1, \varphi), k \in N$, then $v_{2 k} \in C(\bar{D})$.

Note that $v_{2 k+1} \in C^{\infty}(\bar{D}), v_{2 k} \in C(\bar{D})$.

Let us determine the functions $w_{k}(\tau, \varphi)$. Rewrite relation (9) as follows:

$$
\sum_{k=0}^{\infty} \mu^{k}\left(\frac{\partial^{2} w_{k}}{\partial \tau^{2}}+c \mu^{2} \frac{\partial w_{k}}{\partial \tau}+c^{2} \mu^{4} \frac{\partial^{2} w_{k}}{\partial \varphi^{2}}-\sqrt{\tau} w_{k}\right)=\sum_{k=0}^{\infty} \mu^{10 k}\left(h_{2 k}(\varphi)+\mu^{2} \frac{h_{2 k+1,3}(\varphi)}{\sqrt{\tau^{3}}}+\mu^{4} \frac{h_{2 k+1,1}(\mu \tau, \varphi)}{\sqrt{\tau}}\right) .
$$

Hence, taking into account (5), we have:

$$
\begin{gathered}
l w_{0} \equiv \frac{\partial^{2} w_{0}}{\partial \tau^{2}}-\sqrt{\tau} w_{0}=h_{0}, w_{0}(0, \varphi)=0, \lim _{\tau \rightarrow \infty} w_{0}(\tau, \varphi)=0, \\
l w_{1}=0, w_{1}(0, \varphi)=\psi_{1}(\varphi)-v_{0}(1, \varphi), \lim _{\tau \rightarrow \infty} w_{1}(\tau, \varphi)=0, \\
l w_{2}=-c \frac{\partial w_{0}}{\partial \tau}+\frac{h_{1,3}(\varphi)}{\sqrt{\tau^{3}}}, w_{2}(0, \varphi)=0, \lim _{\tau \rightarrow \infty} w_{2}(\tau, \varphi)=0, \\
l w_{3}=-c \frac{\partial w_{1}}{\partial \tau}, w_{3}(0, \varphi)=0, \lim _{\tau \rightarrow \infty} w_{3}(\tau, \varphi)=0, \\
l w_{4}=-c \frac{\partial w_{2}}{\partial \tau}-c^{2} \frac{\partial^{2} w_{0}}{\partial \varphi^{2}}+\frac{h_{1,1}(\tau \mu, \varphi)}{\sqrt{\tau}}, w_{4}(0, \varphi)=0, \lim _{\tau \rightarrow \infty} w_{4}(\tau, \varphi)=0, \\
l w_{5}=-c \frac{\partial w_{3}}{\partial \tau}-c^{2} \frac{\partial^{2} w_{1}}{\partial \varphi^{2}}, w_{5}(0, \varphi)=0, \lim _{\tau \rightarrow \infty} w_{5}(\tau, \varphi)=0, \\
l w_{6}=-c \frac{\partial w_{4}}{\partial \tau}-c^{2} \frac{\partial^{2} w_{2}}{\partial \varphi^{2}}, w_{6}(0, \varphi)=-v_{1}(1, \varphi), \lim _{\tau \rightarrow \infty} w_{6}(\tau, \varphi)=0, \\
l w_{5 k+1}=-c \frac{\partial w_{j-2}}{\partial \tau}-c^{2} \frac{\partial^{2} w_{j-4}}{\partial \varphi^{2}}, w_{j}(0, \varphi)=0, \lim _{\tau \rightarrow \infty} w_{j}(\tau, \varphi)=0, j=7,8,9, \\
l w_{10 k}=-c \frac{\partial w_{10 k-2}}{\partial \tau}-c^{2} \frac{\partial^{2} w_{5 k-3}}{\partial \varphi^{2}}, w_{5 k+1}(0, \varphi)=-v_{k}(1, \varphi), \lim _{\tau \rightarrow \infty} w_{5 k+1}(\tau, \varphi)=0, \\
\partial \varphi_{10 k+2}=-c \frac{\partial w_{10 k}}{\partial \tau}-c^{2} \frac{\partial^{2} w_{10 k-2}}{\partial \varphi^{2}}+\frac{h_{2 k+1,3}(\varphi)}{\sqrt{\tau^{3}}}, w_{10 k+2}(0, \varphi)=0, \lim _{\tau \rightarrow \infty} w_{10 k+2}(\tau, \varphi)=0, w_{10 k}(0, \varphi)=0, \lim _{\tau \rightarrow \infty} w_{10 k}(\tau, \varphi)=0, \\
\frac{\partial w_{10 k+2}}{\partial \tau}-c^{2} \frac{\partial^{2} w_{10 k}}{\partial \varphi^{2}}+\frac{h_{2 k+1,1}(\tau \mu, \varphi)}{\sqrt{\tau}}, w_{10 k+4}(0, \varphi)=0, \lim _{\tau \rightarrow \infty} w_{10 k+4}(\tau, \varphi)=0
\end{gathered}
$$

Let us prove the following statement.

Lemma. The problem

$$
z^{\prime \prime}(t)-\sqrt{t} z(t)=\frac{c}{\sqrt{t^{k}}}, t \in(0, \infty), k=0,1,3, z(0)=z^{0}, \lim _{t \rightarrow \infty} z(t)=0,
$$

has a unique solution (here $c, z^{0}$ are constants).

Proof. We know that the corresponding linear homogeneous differential equation of the second or$\operatorname{der} \tilde{z} "(t)-\sqrt{t} \tilde{z}(t)=0$ has two independent solutions:

$$
z_{1}(t)=\sqrt{t} I_{2 / 5}\left(4 t^{5 / 4} / 5\right), \quad z_{2}(t)=\sqrt{t} K_{2 / 5}\left(4 t^{5 / 4} / 5\right),
$$

where $I_{2 / 5}(s), K_{2 / 5}(s)$ are the modified Bessel functions. 
Solutions to the homogeneous equation have the following property:

$z_{1}(t)=O\left(t^{-\frac{1}{8}} e^{\frac{4}{5} t^{5 / 4}}\right), z_{2}(t)=O\left(t^{-\frac{1}{8}} e^{-\frac{4}{5} t^{5 / 4}}\right), t \rightarrow \infty, z_{1}(0)=0, z_{2}(0) \neq 0, W\left(z_{1}, z_{2}\right)=\frac{1}{c_{1}}, 0 \neq c_{1}=$ const .

The solution to the inhomogeneous equation with the corresponding boundary conditions can be represented as

$$
z(t)=\frac{z^{0} z_{2}(t)}{z_{2}(0)}+c c_{1}\left(z_{2}(t) \int_{0}^{t} s^{-k / 2} z_{1}(s) d s+z_{1}(t) \int_{t}^{\infty} s^{-k / 2} z_{2}(s) d s\right) .
$$

The asymptotic behavior of the solutions $z_{1}(t)$ and $z_{2}(t)$ to the homogeneous equation implies that $z(t)=O\left(t^{-(k+1) / 2}\right)$ when $t \rightarrow \infty$, and $z(t)=O\left(t^{2-k / 2}\right), k=0,1,3$, when $t \rightarrow 0$. This completes the proof of Lemma.

By virtue of Lemma, there exist unique solutions to boundary value problems (11)-(22). It follows from the properties of $z_{1}(t)$ and $z_{2}(t)$ that $w_{10 k}(t)=O\left(1 / t^{1 / 2}\right), w_{10 k+2}(t)=O\left(1 / t^{2}\right), w_{10 k+4}(t)=O(1 / t), t \rightarrow \infty$, and the remaining solutions $w_{n}(t)$ are exponentially small when $t \rightarrow \infty$.

Let us consider boundary value problem (10), (6). Since $\alpha=\lambda t /(a-1), 0<\alpha<1$, then the following decomposition takes place:

$$
\sqrt{1-\alpha}=1+\sum_{j=1}^{\infty} \frac{1}{j !} \frac{1}{2}\left(\frac{1}{2}-1\right) \ldots\left(\frac{1}{2}-j+1\right)(-\alpha)^{j} .
$$

Therefore, homogeneous differential equation (10) can be written in the following form:

$$
\begin{aligned}
& \frac{\partial^{2} z_{0}}{\partial t^{2}}-\sqrt{a-1} z_{0}=0, \quad(t, \varphi) \in D_{1}, z_{0}(0, \varphi)=\psi_{2}(\varphi)-v_{0}(a, \varphi), \lim _{t \rightarrow \infty} z_{0}(\mathrm{t}, \varphi)=0, \\
& \frac{\partial^{2} z_{k}}{\partial t^{2}}-\sqrt{a-1} z_{k}=G_{k}\left(z_{0}, \frac{\partial z_{0}}{\partial t}, \frac{\partial^{2} z_{0}}{\partial \varphi^{2}}, \ldots, z_{k-1}, \frac{\partial z_{k-1}}{\partial t}, \frac{\partial^{2} z_{k-1}}{\partial \varphi^{2}}, \mathrm{t}\right),(t, \varphi) \in D_{1},
\end{aligned}
$$

and the boundary conditions can be represented as $z_{2 n}(0, \varphi)=-v_{n}(a, \varphi), z_{2 n-1}(0, \varphi)=0, \lim z_{k}(\mathrm{t}, \varphi)=0$, where the functions $G_{k}\left(z_{0}, \frac{\partial z_{0}}{\partial t}, \frac{\partial^{2} z_{0}}{\partial \varphi^{2}}, \ldots, z_{k-1}, \frac{\partial z_{k-1}}{\partial t}, \frac{\partial^{2} z_{k-1}}{\partial \varphi^{2}}, \mathrm{t}\right)$ depend linearly on the previous solutions and on the derivatives of these solutions, i.e. on $z_{k-1}, \frac{\partial z_{k-1}}{\partial t}, \frac{\partial^{2} z_{k-1}}{\partial \varphi^{2}}$ and on the variable $t$.

These problems have unique solutions, which decrease exponentially when $t \rightarrow \infty$ :

$$
z_{0}(t, \varphi)=\left(\psi_{2}(\varphi)-\mathrm{v}_{0}(a, \varphi)\right) e^{-\sqrt[4]{a-1} t}, z_{k}(t, \varphi)=e^{-\sqrt[4]{a-1} t} P_{k}(t, \varphi), P_{k} \in C^{\infty}\left(D_{1}\right) .
$$

We have determined all terms of formal asymptotic solution (4). Let us estimate the remainder of this expansion.

Let

$$
u(\rho, \varphi, \varepsilon)=V_{2 n+1}(\rho, \varphi, \varepsilon)+Z_{4 n+2}(t, \varphi, \lambda)+W_{10 n+6}(\tau, \varphi, \mu)+R_{2 n+1}(\rho, \varphi, \varepsilon)
$$
where $V_{2 n+1}(\rho, \varphi, \varepsilon)=\sum_{k=0}^{2 n+1} \varepsilon^{k} v_{k}(\rho, \varphi), \quad Z_{4 n+2}(t, \varphi, \lambda)=\sum_{k=0}^{4 n+2} \lambda^{k} z_{k}(t, \varphi), \quad W_{10 n+6}(\tau, \varphi, \mu)=\frac{1}{\mu} \sum_{k=0}^{10 n+6} \mu^{k} w_{k}(\tau, \varphi)$, $R_{2 n+1}(\rho, \varphi, \varepsilon)$ is the remainder of the series.

Then we obtain the following problem for the residual function $R_{n}(\rho, \varphi, \varepsilon)$ :

$$
\begin{aligned}
& \varepsilon \Delta R_{2 n+1}(\rho, \varphi, \varepsilon)-\sqrt{\rho-1} R_{2 n+1}(\rho, \varphi, \varepsilon)=O\left(\varepsilon^{2 n+2}\right), \varepsilon \rightarrow 0, \quad(\rho, \varphi) \in D, \\
& \mathrm{R}_{2 n+1}(1, \varphi, \varepsilon)=O\left(e^{-1 / \sqrt{\varepsilon}}\right), R_{2 n+1}(\mathrm{a}, \varphi, \varepsilon)=O\left(\varepsilon^{2 m+2}\right), \varepsilon \rightarrow 0, \varphi \in[0,2 \pi] .
\end{aligned}
$$

It is impossible to apply the maximum principle directly, since $\sqrt{\rho-1} \geq 0$ for $1 \leq \rho \leq a$. Therefore, first of all, we replace $R_{2 n+1}(\rho, \varphi, \varepsilon)=\left(a-\rho^{2} / 2\right) r_{2 n+1}(\rho, \varphi, \varepsilon)$.

Then problem (23)-(24) takes the form: 


$$
\begin{gathered}
\varepsilon \Delta r_{2 n+1}-\frac{4 \varepsilon \rho}{2 a-\rho^{2}} \frac{\partial r_{2 n+1}}{\partial \rho}-\left(\sqrt{\rho-1}+\frac{4 \varepsilon}{2 a-\rho^{2}}\right) r_{2 n+1}=O\left(\varepsilon^{2 n+2}\right),(\rho, \varphi) \in D, \\
r_{2 n+1}(1, \varphi, \varepsilon)=O\left(e^{-1 / \sqrt{\varepsilon}}\right), \quad r_{2 n+1}(a, \varphi, \varepsilon)=O\left(\varepsilon^{2 m+2}\right), \varepsilon \rightarrow 0, \varphi \in[0,2 \pi] .
\end{gathered}
$$

For this problem, the maximum principle [3] can be applied. As a result, we obtain an asymptotic estimation: $r_{2 n+1}(\rho, \varphi, \varepsilon)=O\left(\varepsilon^{2 m+1}\right), \varepsilon \rightarrow 0,(\rho, \varphi) \in \bar{D}$.

Therefore, $R_{2 n+1}(\rho, \varphi, \varepsilon)=O\left(\varepsilon^{2 m+1}\right), \varepsilon \rightarrow 0,(\rho, \varphi) \in \bar{D}$.

This completes the proof of Theorem.

\section{References}

1. Gie Gung-Min, Jung Chang-Yeol, Temam R. Recent progresses in boundary layer theory. Discrete \& Continuous Dynamical Systems - A, 2014, Vol. 36, no. 5, pp. 2521-2583. DOI: $10.3934 /$ dcds.2016.36.2521

2. Il'in, A.M. Asimptoticheskie metody $v$ analize (Asymptotic methods in analysis). Moscow, Fizmatlit Publ., 2009, 248 p. (in Russ.).

3. Gilbarg D., Trudinger, N.S. Elliptic partial differential equations of second order. Second edition. Grundlehren der Mathematischen Wissenschaften (Fundamental Principles of Mathematical Sciences), 224. Springer-Verlag, Berlin, 1983, 513 p.

4. Tursunov D.A., Erkebaev U.Z. Asymptotic expansion of the solution of the Dirichlet problem for a ring with a singularity on the boundary. Tomsk State University Journal of Mathematics and Mechanics, 2016, no. 1(39), pp. 42-52. (in Russ.). DOI: 10.17223/19988621/39/5

5. Tursunov D.A., Erkebaev U.Z. Asymptotic expansions of solutions to Dirichlet problem for elliptic equation with singularities. Ufa Mathematical Journal, 2015, Vol. 8, no. 1, pp. 97-107. DOI: $10.13108 / 2016-8-1-97$

6. Tursunov D.A. The generalized boundary function method for bisingular problems in a disk, Trudy Inst. Mat. i Mekh. UrO RAN, 2017, Vol. 23, no. 2, pp. 239-249. DOI: 10.21538/0134-4889-201723-2-239-249

Received November 8, 2019

Bulletin of the South Ural State University Series "Mathematics. Mechanics. Physics" 2020, vol. 12, no. 3, pp. 41-47

Удк 517.955.8

DOI: $10.14529 / \mathrm{mmph} 200306$

\section{АСИМПТОТИЧЕСКОЕ РЕШЕНИЕ ВОЗМУЩЕННОЙ ПЕРВОЙ КРАЕВОЙ ЗАДАЧИ С НЕГЛАДКИМ КОЭФФИЦИЕНТОМ}

\section{Д.А. Турсунов, М.О. Орозов}

Ошский государственный университет, г. Ош, Киргизская Респу́блика

E-mail:d osh@rambler.ru

Рассматривается неоднородная первая краевая задача, т. е. задача Дирихле в кольце для линейного неоднородного эллиптического уравнения второго порядка с двумя независимыми переменными, содержащего малый параметр перед лапласианом. Потенциал уравнения не является гладкой функцией в исследуемой области. Решение исследуемой первой краевой задачи существует и единственно. Явное решение первой краевой задачи построить невозможно. Нас интересует влияние малого параметра на решение задачи Дирихле в рассматриваемой области, когда малый параметр стремится к нулю. Поэтому требуется построить асимптотическое решение первой краевой задачи в кольце. Исследуемая задача имеет две сингулярности (бисингулярная задача): присутствие малого параметра перед лапласианом и решение соответствующего невозмущенного уравнения не является гладкой функцией в рассматриваемой области. Для построения асимпто- 
тического решения применяем модифицированный метод пограничных функций, так как классический метод пограничных функций применить невозможно. Для начала строим формальное асимптотическое решение по малому параметру, а потом оцениваем остаточный член асимптотического разложения. В результате нами построено полное равномерное асимптотическое разложение решения первой краевой задачи в кольце по малому параметру. Построенный ряд решения первой краевой задачи является асимптотическим в смысле Эрдей.

Ключевые слова: задача Дирихле для кольиа; бисингулярная задача; асимптотика; оператор Лапласа; малый параметр.

\section{Литература}

1. Gie, Gung-Min Recent progresses in boundary layer theory / Gung-Min Gie, Chang-Yeol Jung, R. Temam // Discrete \& Continuous Dynamical Systems - A. - 2014. - Vol. 36, no. 5. - P. 2521-2583.

2. Ильин, А.М. Асимптотические методы в анализе / А.М. Ильин, А.Р. Данилин. - М.: Физматлит, 2009. - 248 с.

3. Гилбарг, Д. Эллиптические дифференциальные уравнения с частными производными второго порядка / Д. Гилбарг, Н. Трудингер. - М.: Наука, 1989. - 463 с.

4. Турсунов, Д.А. Асимптотическое разложение решения задачи Дирихле для кольца с особенностью на границе / Д.А. Турсунов, У.З. Эркебаев // Вестник Томского государственного университета. - 2016. - Т. 1(39). - С. 42-52.

5. Tursunov, D.A. Asymptotic expansions of solutions to Dirichlet problem for elliptic equation with singularities / D.A. Tursunov, U.Z. Erkebaev // Ufa Mathematical Journal. - 2015. - Vol. 8, no. 1.P. 97-107.

6. Турсунов, Д.А. Обобщенный метод погранфункций для бисингулярных задач в круге / Д.А. Турсунов // Тр. ИММ УрО РАН. - 2017. - Т. 23, № 2. - С. 239-249.

Поступила в редакцию 8 ноября 2019 2. 